\title{
Acoso psicológico en el trabajo: revisión de la literatura y nuevas líneas de investigación
}

\section{Workplace bullying: literature review and new research avenues}

\author{
Alfredo Rodríguez-Muñoz \\ Departamento de Psicología Social. Facultad de Psicología, Universidad Complutense de Madrid. España.
}

\section{Correspondencia:}

Alfredo Rodríguez Muñoz

Dpto. Psicología Social. Facultad de Psicología

Universidad Complutense de Madrid, Campus de Somosaguas

28223 Madrid. España.

Tfno.: + 34913942935

E-mail.: alfredo.rodriguez@psi.ucm.es

Resumen

Durante los últimos años, se han llevado a cabo un gran número de investigación en el área del acoso psicológico en el trabajo. El presente trabajo pretende realizar una revisión exhaustiva de la literatura sobre acoso laboral. La primera parte del artículo se centra en las diferentes definiciones de acoso y sus tasas de prevalencia. La segunda parte describe los hallazgos empíricos sobre los antecedentes y las consecuencias asociadas al acoso. Por último, se propone una agenda de investigación futura, destacando las lagunas en la investigación del área.

Palabras claves: Acoso psicológico en el trabajo, Salud Laboral; Revisión de la literatura.

\begin{abstract}
||||||||||||||||||||||||||||||||||||||||||||||||||||||||||||||||||||||||||||||||||||||||||||||||||||||||||||||||||||||||||||||||||||||||||||||||||||||||||||||||||||||||||||||||||||||||||||||||||||||||||||||||||||||||||||||||||||||

A great deal of research has been conducted over last years in the field of workplace bullying. The present paper attempts to provide a comprehensive literature review of bullying at work. The first part of the paper is focused on different bullying definitions and its prevalence rates. The second part describes research findings on antecedents and outcomes associated with bullying. Finally, we propose an agenda for future research, highlighting the gaps in investigation in this area.
\end{abstract}

Key words: Workplace bullying; Occupational health; Literature review. 


\section{INTRODUCCIÓN}

En los últimos años, el acoso psicológico en el trabajo está siendo reconocido internacionalmente como un serio problema tanto en el ámbito laboral como en el social. De ser un tema tabú en las organizaciones ha pasado a ser descrito por algunos autores como el tópico de investigación de finales de los años $90^{1}$. Esta preocupación, se ha reflejado en la presencia pública de las cuestiones relacionadas con el acoso laboral, con la consiguiente popularización del término. Es más, el número creciente de casos aparecidos en la prensa ha aumentado el interés social hacia el problema y la preocupación legal por el tema, hasta el punto que ya existen sentencias que consideran el acoso psicológico como un delito penal (Juzgado de lo Penal $\mathrm{n}^{\circ} 1$ de Jaén, septiembre de 2006).

El concepto de acoso psicológico en el trabajo procede de la traducción del anglicismo "mobbing", que deriva del termino inglés "mob" cuyo significado en castellano sería el de una multitud excitada que rodea o asedia a alguien o a algo, bien sea de forma amistosa o bien de forma hostil. En nuestro país este es el término que más se ha extendido y popularizado, aunque la Real Academia Española (RAE) recomienda que sea sustituido por el equivalente español acoso laboral. Sin embargo, la expresión "acoso psicológico" puede parecer la más adecuada. Aunque acoso laboral parece correcto, no introduce la connotación psicológica, por lo que puede inducir a considerar que es algo indirecto, sutil o muy parcial, cuando por el contrario el fenómeno describe conductas de marginación, discriminación y vejación que pueden ser abiertas, dirigidas a la totalidad de la persona, a su hundimiento humano como persona.

En el ámbito internacional, el fenómeno ha sido estudiado bajo distintos términos como "mobbing" " "workplace bullying" ${ }^{1}$, hostigamiento ${ }^{3}$, acoso moral ${ }^{4}$, abuso emocional , persecución ${ }^{6}$ y terror psicológico ${ }^{7}$. A pesar de la variedad conceptual, todos los términos comparten características comunes. En primer lugar, el elemento común a las definiciones es la manifestación de comportamientos negativos de modo repetido y duradero. Aunque no existe un criterio de corte claro, la exposición a las conductas negativas durante aproximadamente seis meses y una vez a la semana ha sido utilizado como una definición operativa para diferenciar entre los casos severos de acoso laboral y la exposición al mismo de forma menos intensa ${ }^{8}$. De esta forma, para concebir una acción como acoso psicológico esta debe cumplir al menos dos criterios temporales: deben tener lugar de manera frecuente (por lo menos una vez a la semana) y durante largo tiempo (por lo menos seis meses) ${ }^{2}$. Así se intentan excluir los conflictos pasajeros y centrados en un momento concreto, que están presentes en todas las organizaciones. Episodios aislados como asignar tareas por debajo de las propias competencias, el ser víctima de bromas por parte de los compañeros ocasionalmente, o el que los compañeros no te avisen para almorzar con ellos, pueden ser vistos como aspectos cotidianos de la vida laboral y no como situaciones de acoso. Sin embargo, tal y como señalan Einarsen y Hauge ${ }^{9}$, las conductas citadas pueden convertirse en actos de acoso cuando ocurren de manera sistemática durante un periodo de tiempo prolongado, dando lugar a un ambiente de trabajo desagradable y hostil para aquel que lo padece.

En segundo lugar, el acoso psicológico no se entiende como un fenómeno estático, sino como un proceso escalar gradual en el que la persona objeto de acoso se enfrenta a agresiones que crecen en intensidad y frecuencia ${ }^{2}$. Matthiesen, Raknes y Røkkum ${ }^{10}$ plantean que el acoso debe ser entendido como un continuo que varía desde la no exposición a una elevada exposición. Por otra parte, numerosos autores plantean que para definir una situación como acoso, es necesario que exista una diferencia de poder, ya sea real o percibida, entre el agresor y la víctima. Las víctimas de acoso a menudo encuentran dificultades para defenderse durante el desarrollo del proceso, lo que implica una diferencia de poder, ya sea real o percibido, entre las partes ${ }^{6}$. Niedl ${ }^{11}$ profundiza en estas diferencias y las clasifica en cuatro grandes bloques: físicas (e.j., corpulencia o fuerza física), psicológicas (e.j., problemas de autoestima de la víctima), económicas (e.j., dependencia económica), y sociales (e.j., diferencia jerárquica en la organización). Estas diferencias impiden que la persona objeto de acoso se defienda. De hecho, en numerosos 
casos el supervisor es el acosador, mientras que los subordinados son los objetivos del acoso, lo que nuevamente indica la existencia de desequilibrios de poder entre las personas implicadas ${ }^{2}$.

Actualmente existe cierto consenso a la hora de delimitar conceptualmente el fenómeno del acoso en el trabajo. Como acoso psicológico se entiende "bostigar, ofender, excluir socialmente a alguien o interferir negativamente en sus tareas laborales. Para concebir una acción como acoso psicológico (o mobbing) esta debe ocurrir regularmente (semanalmente) y durante un prolongado periodo de tiempo (por lo menos seis meses). El acoso es un proceso gradual, durante el cual la persona, desde una posición inferior, se convierte en el objetivo de comportamientos sociales negativos de forma sistemática. Un conflicto no puede ser entendido como una situación de acoso si se trata de un único incidente aislado o si ambas partes en conflicto tienen una "fuerza similar" (p.15)". Una definición similar la ofrece la investigadora finlandesa Denise Salin, quien define el acoso como "comportamientos negativos repetidos y persistentes hacia uno o más individuos, que implica un desequilibrio de poder y crea un ambiente de trabajo bostil" ${ }^{12}$ (p. 1214). En la misma línea lo define el protocolo de actuación sobre el acoso laboral en la Administración Pública, que no considera casos de acoso psicológico aquellas situaciones que ocurran desde relaciones simétricas.

Las acciones o conductas encaminadas a hostigar a una persona suelen ser muy variadas, desde formas explicitas como ataques físicos o verbales, hasta formas más discretas y sutiles, como la exclusión o el aislamiento de la víctima del grupo ${ }^{8}$. En base a análisis teóricos y psicométricos se ha intentado establecer la estructura del fenómeno del acoso psicológico en el trabajo. Por ejemplo, Zapf ${ }^{13}$, tras el análisis del cuestionario LIPT (Leymann Inventory of Psychological Terror), encontró cinco categorías básicas entre las conductas de acoso: 1) acoso relacionado con el trabajo, que dificulta la realización de las tareas; 2) aislamiento social; 3) ataques personales a la vida privada mediante ofensas o insultos; 4) amenazas verbales; 5) violencia física. Por su parte, mediante análisis factoriales del Negative Acts Questionnaire (NAQ) Einarsen y Raknes ${ }^{6}$ encontraron tres componentes: acoso dirigido al ámbito personal, acoso laboral y aislamiento social. Otros autores como Moreno-Jiménez, Rodríguez-Muñoz, Martínez y Gálvez ${ }^{14}$, identificaron dos factores; un componente relacionado con conductas de acoso dirigidas al ámbito personal y privado, y otro componente relativo a las conductas de acoso relacionadas con el trabajo.

Dependiendo si el acosador es un superior o superiores, o si es uno o varios compañeros, las conductas de acoso elegidas pueden variar ${ }^{1,15}$. Los agresores en el mismo nivel en la jerarquía laboral (compañeros), suelen elegir estrategias como la difusión de rumores, los ataques a la vida privada, la burla, y la desigualdad, si tienen en su mano el reparto de tareas o los horarios de trabajo. El uso preferente de estas estrategias se basa en que los compañeros tienen información de la víctima que no suele tener la dirección, circunstancias personales, situación económica, etc. Los acosadores de nivel superior en la jerarquía, prefieren la manipulación hostil y negativa de la comunicación, y el aislamiento social de la víctima, a todos sus niveles. Igualmente, la desigualdad o inequidad es una estrategia muy usada, ya que los superiores tienen casi siempre el poder de decisión de elementos de trabajo como horarios turnos, reparto de tareas, etc. Las agresiones verbales también suelen ser más realizadas por los jefes, en vez de los compañeros, ya que al estar la víctima en un plano (laboral) inferior, siente que es más difícil defenderse.

\section{PREVALENCIA Y EXTENSIÓN DEL PROBLEMA}

Respecto a la prevalencia del acoso, los resultados obtenidos en los distintos estudios deben ser considerados con cautela, ya que existe una amplia variabilidad en las tasas. Los datos existentes varían dependiendo de la definición del acoso, y de las fuentes y métodos utilizados en la recolección de los datos ${ }^{16}$. Algunos estudios han facilitado una 
definición de acoso a los quienes deben indicar si se consideran víctimas de acoso psicológico de acuerdo con la definición dada ${ }^{17}$. En otras investigaciones, los participantes deben indicar si han estado expuestos a determinadas conductas que son consideradas típicas de acoso. Aquellos que respondan haber padecido alguno de los citados comportamientos de manera frecuente (por lo menos una vez a la semana) y durante largo tiempo (por lo menos seis meses), pueden ser considerados víctimas u objeto de acoso $^{6}$. Actualmente se han desarrollado nuevos métodos para discriminar entre los distintos niveles de exposición a conductas de acoso, basados principalmente en técnicas estadísticas como el análisis de cluster latentes ${ }^{18}$. Sin embargo, estos procedimientos no están del todo extendidos, y aún continua existiendo una enorme divergencia en la estimación del problema.

Las primeras investigaciones desarrolladas estimaban, ya en los años ochenta, una prevalencia de un 3.5\% según los estudios pioneros realizados por el profesor Leymann ${ }^{19}$ de la Universidad de Estocolmo. Un estudio finlandés entre trabajadores de universidad halló una prevalencia cercana al $16 \%{ }^{3}$. Por su parte, Hoel y cols..$^{15}$ hallaron que alrededor del 10\% de la población trabajadora de Reino Unido habían padecido acoso laboral. Sin embargo, otras investigaciones han establecido prevalencias más elevadas como un $26.6 \%{ }^{20}$. Sin embargo, los datos más fiables hasta la fecha son los ofrecidos por Zapf, Escartín, Einarsen, Hoel, y Vartia ${ }^{21}$ quienes estimaron recientemente que la prevalencia del acoso con un criterio restrictivo (por lo menos una vez a la semana y durante al menos seis meses) varía entre un 2 y un $4 \%$. Los casos menos severos de acoso ocurren con una mayor frecuencia, alrededor del $10 \%$ de la población europea parece verse afectado por conductas de acoso de forma ocasional.

A nivel nacional, la V Encuesta de Condiciones Trabajo halló una prevalencia del 2.8\% de trabajadores que dicen ser objeto de conductas de acoso diariamente o al menos una vez por semana. La VI Encuesta de Condiciones Trabajo del 2008 señala tasas de afectados sensiblemente inferiores, el $1.4 \%$. Otra fuente de datos importantes sobre prevalencia de acoso aparece recogidas en el primer número monográfico sobre acoso psicológico en España ${ }^{22}$. Las investigaciones incluidas en el mismo informaron prevalencias de afectación en un rango de $9,2 \%$ al $18,9 \%$. Estas tasas de prevalencia difieren de las encontradas en otros países europeos. Comparados con las investigaciones previas, las tasas españolas fueron considerablemente más altas que las halladas en Finlandia ${ }^{17}$, Noruega ${ }^{6}$, Suecia ${ }^{7}$ y el Reino Unido ${ }^{15}$. A pesar de las posibles diferencias, lo que sí parece claro es que el acoso psicológico no se trata de un fenómeno marginal sino con una presencia nada desdeñable y, lo que es más importante, parece que las cifras conocidas son sólo la parte visible de un fenómeno de mayor envergadura. En la tabla 1 aparece un resumen de las distintas tasas de prevalencia y criterios utilizados en diversos estudios realizados en nuestro país.

Tabla 1. Prevalencia del acoso psicológico en distintos estudios nacionales

\begin{tabular}{llrrr}
\hline \multicolumn{1}{c}{ Referencia } & \multicolumn{1}{c}{ Muestra } & N & Definición & Prevalencia \\
\hline Gil-Monte et al. (2006) & Trabajadores de un centro discapacitados & 696 & $1 \mathrm{~b}+2 \mathrm{a}$ & $12,3 \%$ \\
González Trijueque y Graña (2009) & Población general trabajadora & 2861 & $1 \mathrm{~b}+2 \mathrm{a}$ & $5,8 \%$ \\
Justicia et al. (2006) & Empleados de universidad & 548 & $1 \mathrm{~b}+2 \mathrm{a}$ & $9,3 \%$ \\
Piñuel y Onate (2006) & Trabajadores de la Comunidad de Madrid & 4250 & 3 & $9,2 \%$ \\
Rodríguez-Muñoz et al. (2006) & Profesionales sector del cartón ondulado & 352 & $1 \mathrm{~b}+2 \mathrm{a}$ & $4,3 \%$ \\
Moreno-Jiménez et al. (2005) & Sector transportes y comunicaciones & 103 & $1 \mathrm{~b}+2 \mathrm{a}+3$ & $26 \%$ \\
V Encuesta Condiciones Trabajo (2004) & Población general trabajadora & 9290 & $1 \mathrm{~b}+2 \mathrm{a}$ & $2,8 \%$ \\
VI Encuesta Condiciones Trabajo (2007) & Población general trabajadora & 11054 & $1 \mathrm{~b}+2 \mathrm{a}$ & $1,4 \%$ \\
\hline
\end{tabular}

Duración del acoso: 1a) durante los últimos seis meses; 1b) más de 6 meses.

La frecuencia de los comportamientos: 3a) al menos una vez por semana; 3b) menos de una vez por semana.

Incluye la apreciación subjetiva por parte de la víctima de considerarse acosado en base a una definición. 


\section{LOS ANTECEDENTES DEL ACOSO}

Actualmente existe un importante volumen de datos empíricos, sobre los que se puede explorar con cierto rigor las causas y antecedentes implicados en el proceso del acoso psicológico. A este respecto, Einarsen y Hauge ${ }^{9}$ afirman que aunque existen distintas formas de conceptualizar y tratar de explicar los procesos de acoso psicológico, que de forma general pueden resumirse en dos modelos complementarios: la explicación desde un modelo que atiende a factores sociales y organizacionales, o centrando la atención en la personalidad, tanto de víctima como del acosador.

\section{FACTORES ORGANIZACIONES Y SOCIALES}

A lo largo de la literatura, uno de los hallazgos más sistemáticos es la capacidad explicativa de los factores psicosociales en la predicción del acoso ${ }^{23}$. Aunque son numerosos los factores que pueden ser englobados en dicha categoría, algunos autores consideran que las variables antecedentes pueden ser resumidas en cuatro grandes bloques: liderazgo, organización del trabajo, cultura corporativa, y entorno socioeconómico ${ }^{1}$.

Uno de los antecedentes más relevantes es el estilo de liderazgo. De los estudios existentes se desprende una clara asociación entre el acoso y el liderazgo. Por ejemplo, se ha señalado que con frecuencia el acoso ocurre como consecuencia de una gestión inadecuada por parte de los supervisores ${ }^{23}$. Por su parte, Leymann ${ }^{2}$ encontró que el acoso estaba más frecuentemente asociado con un estilo débil de liderazgo. En esta línea, diversos autores ${ }^{24,25}$ han señalado que el estilo de liderazgo "laissez-faire" o pasivo se relacionaba positivamente el acoso, y que además fortalecía la relación entre ciertas variables psicosociales y el acoso. Estos resultados muestran que el estilo "laissez-faire" no es un tipo de liderazgo neutro, sino más bien una forma destructiva de liderazgo que crea un entorno estresante donde el acoso puede prosperar con mayor facilidad. Las formas autoritarias de liderazgo también se han relacionado con la ocurrencia del acoso. A este respecto, Ashforth (1994) hablaba del pequeño tirano o el líder autoritario como antecedente del acoso, y argumentaba que los mandos medios corrían un mayor riesgo de "convertirse" en pequeños tiranos debido a su necesidad de control, su desconfianza en los subordinados y su necesidad de aprobación por la alta dirección. La explicación más directa propone que si los sujetos padecen un liderazgo rígido o inexistente, la organización no será eficaz en la aplicación de los límites marcados por la disciplina, no ofrecerá alternativas para la resolución de conflictos y estará dominada por valores contradictorios. Esto puede dar lugar a ambigüedad de rol y a mayores niveles de estrés, lo que a su vez favorecerá un incremento en las conductas de acoso. Los datos expuestos inducen a considerar que el estilo de liderazgo desempeña un papel decisivo en la aparición del acoso laboral.

La organización del trabajo es otro de los factores cruciales para comprender el fenómeno del acoso. Esta aproximación al problema sitúa su origen etiológico en los factores derivados de la organización del trabajo, planteando que el acoso en numerosas ocasiones es una consecuencia directa de ciertos ambientes laborales. En términos generales, los entornos con mayores tasas de acoso se caracterizan por un elevado nivel de estrés y competitividad ${ }^{23}$. Por ejemplo, en un estudio finlandés, las víctimas y aquellos que habían presenciado situaciones de acoso psicológico describieron su ambiente laboral como estresante, con una alta sobrecarga de trabajo, donde no era posible expresar ideas y opiniones y donde los trabajadores tenían un bajo control sobre las tareas que desempeñaban ${ }^{17}$. De modo similar, y basándose en un largo número de entrevistas con víctimas de acoso, Leymann ${ }^{2}$ concluyó que el acoso estaba fuertemente relacionado con un entorno laboral donde los roles y las estructuras de poder resultaban poco claras.

Desde esta perspectiva, distintos meta-análisis han puesto de manifiesto que tanto la justicia organizacional como el conflicto de rol son dos de los predictores más 
importantes del acoso en el trabajo ${ }^{26,27}$. En esta línea, Messeguer, Soler, García-Izquierdo, Saéz y Sánchez ${ }^{28}$, encontraron que los factores psicosociales que más se relacionan con el acoso eran la definición de rol y la carga mental. Del mismo modo, Rodríguez-Muñoz et $\mathrm{al}^{24}$ hallaron que la justicia procedimental, entendida como la equidad en los procesos para la asignación de recursos y la toma de decisiones y a su aplicación de forma consistente y libre de sesgos, era un potente predictor del acoso laboral. Una posible explicación sobre la relación entre los estresores laborales y el acoso es que los factores organizacionales pueden causar estrés laboral, el cual a su vez puede incrementar el riesgo de conflictos o acoso. El estrés y la frustración pueden, del mismo modo, conducir a la búsqueda de chivos expiatorios dentro de la organización, como un método para descargar la tensión y el estrés ${ }^{28}$.

Otro de los factores que muestran una gran incidencia en la aparición de conductas de acoso es la cultura corporativa de las organizaciones. De acuerdo con Moreno-Jiménez, Rodríguez-Muñoz, Garrosa y Morante ${ }^{29}$ el entorno de trabajo puede transmitir ciertas actitudes y valores a través de la cultura de la organización, lo que puede facilitar la aparición del acoso. La cultura corporativa podría definirse como el conjunto de significados compartidos dentro de una organización. En las organizaciones donde existe una cultura muy fuerte, puede existir mayor conformidad con las normas y las consignas de los superiores, lo que puede facilitar la aparición del acoso psicológico, ya que aquellos miembros que rompen las normas y reglas del grupo podrían estar más expuestos a situaciones de este tipo. De esta forma, parece que en ciertas organizaciones y entornos laborales (militares, bomberos), donde la cultura organizacional es rígida, el acoso, mediante procesos de socialización, puede llegar a institucionalizarse y convertirse en una práctica común, o como señalan Hoel, Rayner y Cooper ${ }^{1}$ en "rituales organizacionales". Al respecto, Topa, Morales y Gallastegui ${ }^{30}$, hallaron en una muestra de trabajadores de emergencias, que la aparición del acoso laboral se encontraba estrechamente relacionado con la cultura organizacional. Algunos estudios han comprobado que existe una mayor probabilidad de aparición del acoso en aquellas organizaciones sin pautas o políticas explicitas respecto al acoso psicológico ${ }^{29}$.

El listado de antecedentes del acoso debe incluir necesariamente variables de orden "macro" como los factores socio-económicos. Por ejemplo, la creciente competitividad en las organizaciones como consecuencia de la globalización económica, ha llevado a que numerosas empresas lleven a cabo reestructuraciones, remodelaciones, fusiones y despidos masivos de personal, con el objetivo de reducir costes. Del mismo modo, la competencia entre trabajadores se ha incrementado debido a las elevadas tasas de desempleo. Por ejemplo, Baron y Neumann ${ }^{31}$ exploraron las relaciones entre diferentes cambios organizacionales y la agresión. Los predictores más potentes de la aparición de conflictos en el lugar de trabajo fueron los siguientes: uso de trabajadores a tiempo parcial, cambios en la dirección y recortes en las remuneraciones de los trabajadores. De igual forma, Muñoz, Guerra, Baron y Munduate ${ }^{32}$ encontraron que el cambio organizacional se relacionaba positivamente con el acoso. De acuerdo con De Witte ${ }^{33}$ los trabajos inseguros y precarios aumentan la presión y la inestabilidad en el grupo de trabajo, lo que puede conducir a la desorganización dando lugar a un incremento en el conflicto y la ambigüedad de rol. A medida que aumenta la inseguridad en el trabajo, es menos probable que los trabajadores hagan frente y desafíen el trato injusto y agresivo por parte de sus jefes. En este mismo sentido se pronuncia la resolución del Parlamento europeo sobre el acoso psicológico en el trabajo (2001/2339/INI), señalando la existencia de un vínculo claro entre el acoso y un trabajo con un alto grado de competitividad, menor estabilidad en el empleo y una situación laboral precaria. A pesar de su dificultad de estudio, no se debe desdeñar el impacto potencial de estos cambios y procesos sobre el acoso.

Tal y como se ha observado, los antecedentes psicosociales muestran un alto nivel explicativo, sin embargo una comprensión global del fenómeno pasa, necesariamente, por analizar el rol de las variables personales, puesto que numerosas características individuales, tanto de las víctimas como de los acosadores, influyen en todo el proceso ${ }^{34}$. 


\section{FACTORES INDIVIDUALES}

Una de las cuestiones que más debate ha suscitado en la investigación sobre acoso y que aún no ha producido resultados definitivos hace referencia al papel de la personalidad. Lo que diversos autores denominan como "hipótesis de la personalidad" hace referencia a aquellos elementos del proceso de acoso que no son explicables exclusivamente por el contexto donde tiene lugar. Al comienzo del estudio del acoso, y probablemente con el objetivo de proteger a las victimas, se eludió estudiar el papel que los factores personales pudieran tener en su curso y aparición. Sólo recientemente han comenzado a estudiarse los factores de riesgo o de vulnerabilidad relacionados con el acoso. No obstante, se debe ser precavido al respecto, puesto que desde esta perspectiva se puede culpar a la víctima de la situación o derivar en una "caza de brujas" ${ }^{34}$. El énfasis no está tanto en la causalidad como en la vulnerabilidad. De acuerdo con Moreno-Jiménez et $\mathrm{al}^{35}$ (p. 374) "el análisis del acoso psicológico desde modelos de personalidad no debe confundir la influencia con la responsabilidad o la culpa, ni con el supuesto de psicopatologías previas".

La evidencia empírica actualmente disponible aporta dos tipos de información relevantes. Por un lado, contamos con un importante volumen de datos e hipótesis referentes a las características de personalidad de aquellos que padecen acoso en el trabajo. En segundo lugar, se han realizado diversos intentos para precisar cuáles serían los atributos psicológicos que podrían caracterizar a los acosadores, aunque en este sentido los datos son comparativamente mucho más escasos y parciales.

\section{El perfil de la víctima}

La hipótesis de la personalidad postula una clara vinculación entre ciertos factores personales y la aparición del acoso en el trabajo. Por ello, se ha hipotetizado que las víctimas de acoso pudieran ser "seleccionadas" debido a sus características de personalidad ${ }^{17}$, debido a que los acosadores los perciben como personas débiles. Desde esta perspectiva se han tratado identificar las variables individuales y de personalidad asociadas con el acoso psicológico. Por ejemplo, algunos autores describen a las víctimas como paranoicas, rígidas, compulsivas ${ }^{28}$, con falta de competencias sociales ${ }^{34}$ y con mayores puntuaciones en neuroticismo ${ }^{17}$. En la misma línea, Smith, Singer, Hoel y Cooper ${ }^{36}$ hallaron una relación significativa entre las experiencias de acoso escolar y las de acoso psicológico en el trabajo. Einarsen et al $^{37}$ encontraron que muchas de las víctimas de acoso en Noruega consideraban que determinadas características de su personalidad como su baja autoestima o timidez contribuía a su problema. También se ha constatado que otras variables de personalidad como el neuroticismo o la autoeficacia se relacionan significativamente con el acoso psicológico ${ }^{17},{ }^{34}$. En esta línea, estudios de meta-análisis han señalado que la autoestima tiene un efecto negativo sobre el acoso ${ }^{27}, \mathrm{y}$ que el afecto negativo correlaciona en mayor medida con el acoso que el afecto positivo ${ }^{26}$.

De forma similar, O'Moore, Seigne, McGuire, y Smith ${ }^{38}$ informan que las víctimas son menos estables emocionalmente, mientras que Einarsen et $\mathrm{al}^{37}$ han hallado que las víctimas de acoso muestran una pobre auto-imagen, al igual que una alta ansiedad en las situaciones sociales. Las víctimas de acoso han sido descritas como excesivamente suspicaces, cognitivamente poco flexibles y con una visión poco realista de sus propias habilidades y recursos ${ }^{28}$. La controversia radica en si estas características deben ser consideradas causas del acoso o si por el contrario son resultado del proceso ${ }^{38}$ (véase apartado futuras líneas de investigación).

\section{El perfil del acosador}

Por lo que respecta a las características de personalidad de las personas que llevan a cabo conductas de acoso, diversos autores plantean que este aspecto constituye una de las grandes incógnitas de la investigación en acoso psicológico ${ }^{39}$. En la mayoría de los estudios existentes, la personalidad de los agresores es evaluada principalmente mediante 
las descripciones realizadas por las víctimas, con lo que la información que se obtiene resulta limitada y sesgada. Las características de personalidad que han sido atribuidas a los agresores son diversas.

Algunas aproximaciones, caracterizadas en ocasiones por planteamientos sensacionalistas, han sugerido un posible perfil de personalidad psicopatológico. Desde esta perspectiva se ha planteado la idea de una personalidad proclive a la violencia. Davenport, Schwatrz, Pursell y Elliott ${ }^{40}$ plantean que el acosador se caracteriza por ser excesivamente controlador, celoso, con ansias de poder y con limitadas competencias sociales. Por su parte, Zapf y Einarsen ${ }^{34}$ han señalado que el acoso psicológico se encuentra estrechamente relacionado con la autoestima del agresor. Una autoestima excesivamente elevada puede conducir a comportamientos tiránicos, al asociarse con un excesivo perfeccionismo, arrogancia y narcisismo. Es más, determinadas situaciones pueden suponer una amenaza a la autoestima, por lo que las personas pueden llevar a cabo comportamientos de acoso como una forma de proteger su autoestima inestable y sus inseguridades ${ }^{2}$. De esta forma, la agresión sería más probable en personas con la autoestima alta, antes que con baja, puesto que las personas con bajos niveles de autoestima muestran síntomas depresivos y retraimiento, más que tendencias agresivas hacia los demás ${ }^{41}$.

Otras características que se asocian con el acosador son las de conformismo y ensalzamiento de la obediencia a la autoridad. Todas estas dimensiones del análisis psicológico, encontramos atributos o propiedades análogas o muy próximas a las que presentan otros tipos de agresores o personalidades violentas. Por ejemplo, la impulsividad y la ausencia de empatía, han sido frecuentemente atribuidas a cierta clase de delincuentes, y a los responsables de maltratos familiares, los violadores, etc.

Matthiesen y Einarsen ${ }^{42}$ introdujeron en la literatura el concepto de víctimas activas. Se refiere a víctimas de acoso con comportamientos activos y agresivos que se caracterizan por un patrón de reacción ansioso-agresivo. Estas personas pueden comportarse de un modo que cause irritación y tensión a su alrededor, corriendo de esta forma un mayor riesgo de ser aislado o excluido socialmente, debido a que los demás perciben su conducta como molesta y agresiva. La investigación de Matthiesen y Einarsen ${ }^{42}$ halló que estas víctimas acosan a los más débiles mientras que al mismo tiempo son acosados por otros superiores o compañeros con más poder.

\section{LOS EFECTOS DEL ACOSO PSICOLÓGICO EN EL TRABAJO}

El acoso psicológico en el trabajo es considerado un severo estresor psicosocial que puede afectar seriamente el funcionamiento cotidiano de aquellos que lo padecen, con la aparición de diversos síntomas de estrés ${ }^{43}$. Además, el acoso también puede tener consecuencias negativas a nivel organizacional. Por ello, algunos autores consideran que estamos ante la principal fuente de estrés social en el trabajo, y un problema más devastador para los empleados afectados que la suma de todos los demás estresores laborales juntos ${ }^{44}$. Veamos algunas de las principales consecuencias de este fenómeno:

\section{Efectos sobre el individuo}

Distintos estudios han puesto de manifiesto que el acoso psicológico se encuentra fuertemente relacionado con diferentes problemas de salud, como por ejemplo síntomas psicosomáticos ${ }^{45}$, depresión ${ }^{44}$, fatiga ${ }^{14}$, problemas de sueño ${ }^{35} \mathrm{y}_{\text {ansiedad }}{ }^{46}$. Basándose en observaciones y entrevistas clínicas a víctimas de acoso, Brodsky ${ }^{28}$ identificó tres patrones de efectos sobre las víctimas. Algunos expresaban sus reacciones mediante el desarrollo de síntomas físicos no excesivamente graves, tales como sensación de debilidad, pérdida de fuerza, fatiga crónica, dolores y jaquecas. Otros reaccionaban con depresión y sintomatología asociada, como sensación de impotencia, falta de autoestima e insomnio. Un tercer grupo reaccionaba con síntomas psicológicos, como la hostilidad, problemas de memoria, hipervigilancia, sentimientos de victimización y evitación de los contactos sociales. 
En vista de la constelación de síntomas señalados, se ha hipotetizado que las víctimas de acoso pueden padecer trastorno por estrés postraumático (TEPT en adelante). Las personas que padecen TEPT experimentan tres clases de síntomas ${ }^{47}$ : a) reexperimentación del hecho traumático, b) evitación y embotamiento psíquico, y c) hiperactivación. Según las investigaciones al respecto, parece que entre las víctimas de acoso la probabilidad de padecer TEPT es elevada. Leymann y Gustafsson ${ }^{48}$ encontraron que el TEPT constituía el diagnóstico adecuado de aproximadamente el $90 \%$ de una muestra de 64 víctimas de acoso. Recientemente, Rodríguez-Muñoz et $\mathrm{al}^{49}$, en un estudio entre víctimas de acoso psicológico en España, hallaron una prevalencia de TEPT que superaba el 40\%. Igualmente, se ha señalado que la gravedad de los síntomas entre las víctimas de acoso puede ser mayor que en víctimas de otros tipos de trauma. Matthiesen y Einarsen ${ }^{50} \operatorname{comparar}_{\text {las }}$ puntaciones obtenidas por un grupo de víctimas de acoso con las de otras muestras expuestas a diversos acontecimientos traumáticos, como personas con familiares involucrados en accidentes de tráfico, personal de la ONU que había estado destinado en zonas bélicas y estudiantes de medicina expuestos a situaciones altamente estresantes (e.j., primera autopsia). Se observó que las víctimas de acoso presentaban puntuaciones más elevadas en sintomatología postraumática respecto a los tres grupos, especialmente en reexperimentación del trauma y en evitación.

Se trata esta de una línea de investigación rodeada de cierta polémica. En el diagnóstico del TEPT la persona debe haber estado expuesta a un acontecimiento estresante y extremadamente traumático que represente un peligro para su vida o cualquier otra amenaza para su integridad física ${ }^{47}$. Este criterio supone un problema en relación al diagnóstico del TEPT en víctimas de acoso, ya que las acciones típicas de acoso son predominantemente agresiones no físicas. Por ello, se han propuesto diagnósticos alternativos para las víctimas de acoso, como por ejemplo el trastorno por estrés agudo o el trastorno adaptativo. El trastorno por estrés agudo se diferencia del TEPT en que el cuadro sintomático del primero debe aparecer y resolverse en las primeras 4 semanas posteriores al acontecimiento traumático. Respecto al trastorno adaptativo el factor estresante puede tener cualquier intensidad. El diagnóstico de trastorno adaptativo es apropiado tanto cuando las respuestas a un desencadenante extremo no reúnen los criterios diagnósticos del TEPT, como cuando el estrés postraumático aparece en respuesta a desencadenantes no excesivamente amenazantes para la integridad física (p.e. abandono del cónyuge). Además, una vez que el estresor haya cesado, la sintomatología no debe durar más de seis meses. Como han puesto de manifiesto la duración de los síntomas postraumáticos en víctimas de acoso excede notablemente los criterios temporales que los trastornos mencionados proponen ${ }^{48}$.

Además, hay que tener en cuenta otros elementos. En primer lugar, y como afirman algunos de los expertos en psicología del trauma, que "la clasificación del trastorno del estrés post traumático sirve mucho más de ayuda para el diagnóstico que como descriptor de la experiencia psicológica de la víctima. Sin duda, una comprensión del trauma significa tener en cuenta la totalidad de las experiencias de los supervivientes" 51 (p. 50). En segundo lugar, Ravin y Boal ${ }^{52}$ han señalado que con frecuencia la experiencia clínica precede al diagnóstico reflejado en el manual. Por otra parte, cuando una persona es acosada en el trabajo, en ocasiones, incluso tras el cese del hostigamiento, las víctimas se enfrentan a amenazas a su identidad personal, social y profesional, y en algunos casos a su capacidad financiera. Por ello, cuando hablamos de trauma causado por la violencia psicológica en el trabajo no estamos, pues, hablando tan sólo de una sintomatología de índole psicopatológica, sino de unos efectos que pueden ir más allá. En este sentido, el significado del agente traumático tiene también una importancia decisiva en las consecuencias. El rápido deterioro de la salud mental en víctimas de acoso se puede explicar, en parte, por la pérdida brusca de aportes psicosociales, como la disponibilidad económica, la seguridad física, la pérdida de habilidades laborales, la imposibilidad de predecir y planificar el futuro y la pérdida de una posición social valorada.

Las secuelas del acoso también pueden extenderse al ámbito familiar y social. Debido al grado de estrés social que provoca el acoso, los afectados pueden sufrir cambios 
en sus patrones de comunicación, y en sus niveles de irritabilidad y negatividad, que a su vez afectará a la calidad de su relación con su red socio-familiar. Los familiares y amigos pueden considerar que la víctima vive el suceso con una preocupación obsesiva. A menudo la víctima se aísla cada vez más, por la sensación de incomprensión de su entorno. En este sentido, numerosas víctimas de acoso indican que carecen de apoyo social $^{44,45}$.

\section{Efectos sobre la organización y el entorno}

Los efectos negativos del acoso no perjudican sólo al individuo, sino que también pueden producir un deterioro en la organización. El primer y más evidente coste es el absentismo y la duración de las bajas del personal. Los trabajadores afectados por acoso psicológico, antes o después, presenta dificultades y problemas de salud que le llevan primero a bajas cortas por enfermedad con las que se intenta recuperarse de algunos problemas de salud experimentados y tratar de reformular la situación. Con el tiempo, se producen bajas de más larga duración, algunas de ellas debido a los problemas psicológicos y médicos mencionados. Diversos estudios han mostrado que el acoso psicológico está asociado con altas tasas de absentismo, numerosos intentos de abandonar la organización y con una alta rotación laboral ${ }^{2,53}$. Se ha comprobado que el acoso es un fuerte predictor de las bajas por enfermedad. En un estudio entre personal sanitario, se estudió que trabajadores tenían un mayor número de bajas medicas certificadas. Se comprobó que, tras controlar el sexo y la edad, las bajas laborales eran un 51\% más frecuente en víctimas de acoso que en el resto de los trabajadores, algunos con otro tipo de problemas médicos y/o psicológicos ${ }^{54}$.

El acoso también se ha relacionado con la disminución de la satisfacción laboral, la motivación en el trabajo, y el compromiso organizacional. Rodríguez-Muñoz et al ${ }^{55}$, en un estudio longitudinal, encontraron que el acoso se asociaba con presentaban menores niveles de satisfacción laboral. Bowling y Beehr (2006) observaron que el acoso se asociaba negativamente con el compromiso organizacional. Resultados similares fueron encontrados por Einarsen, Matthiesen y Skogstad ${ }^{56}$, quienes hallaron relaciones significativas entre las experiencias de acoso y los síntomas de burnout.

Otra de las consecuencias son la disminución de la cantidad y calidad del trabajo desarrollado por la persona afectada, el entorpecimiento o la imposibilidad del trabajo en grupo, problemas en los circuitos de información y comunicación, etc. Es posible también, en fases más avanzadas, que el trabajador afectado abandone la empresa. Cuando se consigue, el abandono de la empresa puede representar una pérdida de experiencia y conocimientos para la organización.

No hay lugar a dudas que los problemas señalados acarrean un alto coste financiero en las organizaciones. Se ha estimado que el coste económico de caso de acoso para una organización varía en entre 30.000 y 100.000 dólares estadounidenses ${ }^{7}$. Los cálculos realizados por Hoel, Einarsen, y Cooper ${ }^{53}$ de un caso de acoso en Gran Bretaña, sitúan los costes alrededor de 28.000 libras británicas. Además, hay que sumarles costes indirectos, como el daño a la imagen pública de las organizaciones, y la consecuente pérdida potencial de clientes.

\section{FUTURAS LÍNEAS DE INVESTIGACIÓN}

Tal y como se ha podido comprobar, el desarrollo de la investigación sobre acoso psicológico ha crecido y evolucionado sustancialmente en los últimos años. Sin embargo, para alcanzar una comprensión completa del fenómeno aún quedan por explorar numerosos aspectos del problema. Veamos algunas posibles futuras líneas de investigación.

Una de las líneas más necesarias se centra en los estudios longitudinales, puesto que la gran mayoría de las investigaciones sobre acoso se han llevado a cabo mediante diseños transversales, lo que no permite concluir en términos causales. Esto hace que se conozca 
poco sobre cómo los distintos elementos involucrados en el proceso de acoso se relacionan entre sí a lo largo del tiempo. Tal y como plantean Rodríguez-Muñoz et al ${ }^{55}$, la dirección de la relación entre los factores se ha inferido desde modelos teóricos, en lugar de basarse en datos empíricos. Por ejemplo, en el caso de las consecuencias del acoso, existe un elevado consenso sobre lo dañino de este fenómeno, siendo considerando como un severo estresor que puede conducir a diversos problemas de salud. Sin embargo, al igual que con el resto de formas de estrés laboral, es posible que existan otras formas de causalidad, como la inversa o la reciproca. En este sentido, Kivimäki et al ${ }^{57}$, encontraron que un incremento en las conductas de acoso se relacionaba con mayores niveles de depresión dos años después, que a su vez contribuía a la aparición de un mayor número de conductas de acoso. De modo similar, en un reciente estudio longitudinal Nielsen et al ${ }^{58}$ hallaron que existía una relación reciproca entre los problemas psicológicos y el acoso. Estos hallazgos evidencia la complejidad del proceso de acoso. Una de las mayores controversias en el campo radica en el papel de la personalidad en el acoso. Actualmente no se sabe con exactitud si la personalidad es un factor de vulnerabilidad para sufrir acoso, o si por el contrario se trata de una consecuencia del mismo. Leymann ${ }^{2}$ se opuso enérgicamente a la idea de que la personalidad de un individuo predisponga a convertirse en objeto de comportamientos de acoso. Este autor planteaba que las características inestables y ansiosas de muchas víctimas deberían ser entendidas como una consecuencia de la experiencia traumática del acoso. No hay duda de que este debate continuará hasta que se lleven a cabo investigaciones longitudinales sobre esta cuestión. Hasta la fecha, no existen estudios longitudinales sobre acoso con población española. Por tanto, parece evidente la necesidad de llevar a cabo este tipo de estudios, para esclarecer la dirección de las relaciones entre el acoso y sus antecedentes y consecuencias.

Dentro del ámbito de las consecuencias asociadas con el acoso, otra prometedora línea de investigación consiste en la inclusión de medidas objetivas. Según diversos autores, para comprender la relación entre los factores de estrés laboral y los problemas de salud resulta de vital importancia el estudio de las variables fisiológicas ${ }^{59}$. Sin embargo, los datos al respecto en el campo del acoso son muy limitados. Tan solo un par de estudios han aportado datos sobre alteraciones del ciclo circadiano en víctimas de acoso, con menores niveles de concentración de cortisol salivar ${ }^{60,61}$. Por su parte, MorenoJiménez et $\mathrm{al}^{62}$ hallaron que el acoso se relacionaba positivamente con la activación fisiológica, evaluada mediante la presión arterial sistólica y diastólica, así como con un mayor índice de masa corporal. Se ha sugerido que una desregulación del eje hipotalámicopituitario-adrenal (HPA) puede explicar los problemas de salud que experimentan las personas en situaciones de estrés crónico ${ }^{63}$. Además, con este tipo de variables se podría "objetivar" en mayor medida el daño que pueden padecer las víctimas de acoso. Por ello, y $\sin$ querer desmerecer la importancia de los indicadores de salud autoinformados, resulta crucial avanzar en este campo.

Por otra parte, la mayoría de las investigaciones se han centrado en analizar la díada victima-acosador, desde una perspectiva individual. Sin embargo, los procesos de acoso psicológico aparecen y se desarrollan en un contexto organizacional y social que los configura y condiciona. Por lo tanto, otro reto en este campo de investigación consiste en superar esa visión individualista y parcial, y explorar el acoso desde enfoques y análisis grupales. Del mismo modo, gran parte de la investigación ha centrado su atención en las personas objeto de las conductas de acoso, dejando a un lado el estudio de los agresores. Existe una creencia, especialmente arraigada entre las víctimas de acoso, sobre la estabilidad del acoso en el tiempo, que hace que los agresores sigan acosando, mientras que las víctimas nunca puedan llevar a cabo este tipo de acciones sobre sus compañeros o superiores. Sin embargo, Bowling y Beehr ${ }^{26}$ plantean que el proceso de acoso dista mucho de un simple patrón emisor-receptor. De hecho, es posible que algunas víctimas puedan convertirse en agresores mediante un mecanismo de agresión desplazada, o por mera venganza. Al respecto, existe evidencia empírica incipiente que apunta hacia esta posibilidad $^{42}$. Por lo tanto, los futuros estudios deberían profundizar en que padecer y llevar a cabo este tipo de conductas pueden ser procesos relacionados. 
Uno de los aspectos que mayor interés suscitan entre la opinión publica es el porcentaje de personas afectadas por esta problemática. Como se ha visto en el apartado sobre prevalencia, su estimación no está exenta de controversia. Como sugieren Escartín et $\mathrm{al}^{64}$, es importante distinguir entre las experiencias subjetivas y objetivas de acoso. Aunque muchas personas pueden creer estar siendo sometidas a conductas de acoso en su trabajo, esta percepción subjetiva no se debe identificar necesariamente con la existencia del mismo. De modo similar, situaciones que pueden ser consideradas ofensivas por algunas personas, pueden ser interpretadas como graves por otras. Como método de evaluación del acoso, numerosos estudios se han basado en la experiencia subjetiva, la cual ha sido criticada por ser excesivamente simple, con poca fiabilidad y validez, y por no recoger la heterogeneidad ofrecida por las distintas conductas de acoso ${ }^{18}$. Actualmente, aún predomina el criterio de Leymann ${ }^{2}$ para determinar si una situación puede ser considerada como acoso. Recordemos que una persona que responda haber padecido alguna de las conductas consideradas como acoso de forma frecuente y durante largo tiempo pueden ser clasificadas como víctimas de acoso. Sin embargo, estos criterios son claramente arbitrarios, puesto que Leymann los desarrolló en función de su experiencia clínica, con un colectivo concreto de víctimas. Por lo tanto, en el futuro habría que intentar unificar y desarrollar los distintos métodos para evaluar el acoso laboral. Esto nos conduciría a una mayor fiabilidad en los datos de prevalencia, así como a una mayor facilidad a la hora de compararlos. Igualmente, sería recomendable que las medidas de autoinforme se complementaran con observadores externos, para analizar el acuerdo entre ambos tipos de información.

Por último, pero no por ello menos importante, es necesario profundizar en las intervenciones en las situaciones de acoso. El estudio del proceso de acoso es relevante, ya que son los elementos que facilitan y permiten posteriormente la selección de criterios de actuación orientados a la prevención e intervención. Sin embargo, en la actualidad apenas existen estudios que evalúen la eficacia de los distintos tipos de intervención para prevenir o actuar contra el acoso. Una de las excepciones más notables la constituye el proyecto Danés "Titijob", orientado a la rehabilitación de víctimas de acoso psicológico. Son especialmente importantes aquellas intervenciones preventivas que inciden en los factores etiológicos, puesto que de esta forma se actúa sobre el acoso de la forma más efectiva, directamente sobre sus causas.

\section{REFERENCIAS BIBLIOGRÁFICAS}

1. Hoel H, Rayner C, Cooper C. Workplace bullying. En: Cooper C, Robertson IT (Eds.). International Review of Industrial and Organizational Psychology, 14. Chichester: Wiley; 1999.

2. Leymann H. The content and development of mobbing at work. Eur J Work Org Psychol 1996;5:165-84.

3. Björkqvist K, Österman K, Hjelt-Bäck M. Aggression among university employees. Aggress Behav. 1994;20:173-84.

4. Hirigoyen MF. El acoso moral en el trabajo. Barcelona: Paidós; 2001.

5. Keashly L. Emotional abuse in the workplace: Conceptual and empirical issues. J Emotional Abuse. 1998;1:85-117.

6. Einarsen S, Raknes BI. Harassment in the workplace and the victimization of men. Violence Vict. 1997; 12:247-63

7. Leymann H. (1990). Mobbing and psychological terror at workplaces. Violence Vict 1990;5:119-26.

8. Einarsen S, Hoel H, Zapf D, Cooper CL. The concept of bullying at work. The European tradition. En Einarsen S. Hoel H, Zapf D, Cooper CL (Eds.). Bullying and Emotional Abuse in the Workplace. International perspectives in research and practice (pp. 3-30). London: Taylor \& Francis; 2003.

9. Einarsen S. Hauge LJ. Antecedentes y consecuencias del acoso psicológico en el trabajo: Una revisión de la literatura. Revista de Psicología del Trabajo y de las Organizaciones 2006;22:251-73.

10. Matthiesen SB, Raknes BI, Røkkum O. Mobbing på arbeidsplassen. Tidsskrift for Norsk Psykologforening 1989; 26:761-74. 
11. Niedl K. Mobbing and well-being: Economic and personnel development implications. Eur J Work Org Psychol 1996;5:239-50.

12. Salin D. Ways of explaining workplace bullying: A review of enabling, motivating and precipitating structures and processes in the work environment. Human Relations 2003;56:1213-32.

13. Zapf D. Organisational, work group related and personal causes of mobbing/bullying at work. Int J Manpower 1999;20:70-85.

14. Moreno-Jiménez B, Rodríguez-Muñoz A, Martínez M, Gálvez M. Assesing workplace bullying: Spanish validation of a shortened version of the negative acts questionnaire. Span J Psychol 2007;10:449-57.

15. Hoel H, Cooper C, Faragher B. The experience of bulling in Great Britain: The impact of organizational status. Eur J Work Org Psychol. 2001;10:443-66.

16. Nielsen MB, Skogstad A, Matthiesen SB, Glasø L, Aasland MS, Notelaers G, et al. Prevalence of workplace bullying in Norway: Comparisons across time and estimation methods. Eur J Work Org Psychol. 2009;18:81-101.

17. Vartia M. The sources of bullying - psychological work environment and organizational climate. Eur J Work Org Psychol. 1996;5:203-14.

18. Notelaers G, Einarsen S, De Witte H, Vermunt JK. Measuring exposure to bullying at work: the validity and advantages of the latent class cluster approach. Work Stress 2006;20:288-301.

19. Leymann H. Vuxenmobbning: om psykiskt våld i arbetslivet. Lund: Studentlitteratur; 1986.

20. Niedl K. Mobbing/bullying am Arbeitsplatz. Eine empirische Analyse zum Phänomen sowie zu personalwirtschaftlich relevanten Effekten von systematischen Feindligkeiten. Doctoral dissertation. München, Germany: Rainer Hampp Verlag; 1995.

21. Zapf D, Escartín J, Einarsen S, Hoel H, Vartia M. Empirical findings on prevalence and risk groups of bullying in the workplace. En Einarsen S. Hoel H, Zapf D, Cooper CL (Eds.), Workplace bullying: Developments in theory, research and practice. London \& New York: Taylor \& Francis; 2011.

22. Moreno-Jiménez B, Rodríguez-Muñoz A. Introducción al número monográfico sobre acoso psicologico en el trabajo: una perspectiva general. Revista de Psicologia del Trabajo y de las Organizaciones 2006;22:245-50.

23. Hoel H. Salin D. Organisational antecedents of bullying at work. En Einarsen S. Hoel H, Zapf D, Cooper CL (Eds.). Bullying and Emotional Abuse in the Workplace. International perspectives in research and practice. London: Taylor \& Francis; 2003.

24. Rodríguez-Muñoz A, Gil F, Moreno-Jiménez B. Factores organizacionales y acoso psicológico en el trabajo: el papel moderador del liderazgo "laissez-faire". Revista Psicología Social En prensa.

25. Skogstad A, Einarsen S, Torsheim T, Aasland MS, Hetland H. The destructiveness of laissez-faire leadership behavior. J Occup Health Psychol 2007;12:80-92.

26. Bowling NA, Beehr TA. Workplace harassment from the victim's perspective. A theoretical and metaanalysis. J Appl Psychol 2006;91:998-1012.

27. Topa G, Depolo M, Morales JF. Acoso laboral: meta-análisis y modelo integrador de sus antecedentes y consecuencias. Psicothema 2007;19:88-94.

28. Brodsky CM. The Harassed Worker, Lexington Books, DC Heath and Company,Toronto; 1976.

29. Moreno-Jiménez B, Rodríguez-Muñoz A, Garrosa E, Morante ME. Antecedentes Organizacionales del acoso psicológico en el trabajo: un estudio exploratorio. Psicothema 2005;17(4):627-32.

30. Topa G, Morales JF, Gallastegui JA. Acoso laboral: relaciónes con la cultura organizacional y los resultados personales. Psicothema 2006;18:766-71.

31. Baron RA, Neuman, JH. Workplace violence and workplace aggression: evidence on their relative frequency and potential causes. Aggress Behav. 1996;22:161-73.

32. Muñoz H, Guerra JM, Baron M, Munduate L. El acoso psicológico desde una perspectiva organizacional. Papel del clima organizacional y los procesos de cambio. Revista de Psicología del Trabajo y de las Organizaciones 2006;22:347-61.

33. De Witte H. Job insecurity: Review of the international literature on definitions, prevalence, antecedents and consequences. SA J Industrial Psychol 2005;31(4):1-6.

34. Zapf D, Einarsen S. Individual antecedents of bullying. En Einarsen S. Hoel H, Zapf D, Cooper CL (Eds.). Bullying and Emotional Abuse in the Workplace. International perspectives in research and practice. London: Taylor \& Francis; 2003.

35. Moreno-Jiménez B, Rodríguez-Muñoz A, Moreno Y, Garrosa E. El papel moderador de la asertividad y la ansiedad social en el acoso psicológico en el trabajo: dos estudios empíricos 2006. Revista de Psicología del Trabajo y de las Organizaciones;22:363-80. 
36. Smith PK, Singer M, Hoel H, Cooper CL. Victimization in the school and the workplace: Are there any links? Br J Psychol 2003;94:175-88.

37. Einarsen S, Raknes BI, Matthiesen SB. Bullying and harassment at work and their relationships to work environment quality: An exploratory study. Eur J Work Org Psychol 1994;4:381-401.

38. Moore M, Seigne E, McGuire L, Smith M. Victims of bullying at work in Ireland. J Occup Health Safety 1998; $14: 568-74$.

39. Rayner C, Cooper CL. The black hole in "bullying at work research". Int J Management decision making 2003; $4: 47-64$

40. Davenport N, Schwatrz D, Pursell R, Elliott G. Mobbing: Emotional abuse in the american workplace. Iowa: Civil Society Publishing; 1999.

41. Baumeister RF, Smart L, Boden JM. Relation of threatened egotism to violence and aggression: The dark side of high self-esteem. Psychol Rev 1996;103:5-33.

42. Matthiesen SB, Einarsen S. Perpetrators and targets of bullying at work: Role stress and individual differences. Violence Vict. 2007;22:735-53.

43. Rodríguez-Muñoz, A. Los efectos del acoso psicológico en el trabajo: el papel de los procesos cognitivos. Tesis Doctoral. Universidad Autónoma de Madrid, 2009

44. Einarsen S, Mikkelsen EG. Individual effects of exposure to bullying at work. En Einarsen S. Hoel H, Zapf D, Cooper CL (Eds.). Bullying and Emotional Abuse in the Workplace. International perspectives in research and practice. London: Taylor \& Francis; 2003.

45. Zapf D, Knorz C, Kulla M. On the relationship between mobbing factors, and job content, social work environment and health outcomes. Eur J Work Org Psychol 1996;5:215-37.

46. Mikkelsen EG, Einarsen S. Bullying in Danish work-life: Prevalence and health correlates. Eur J Work Org Psychol 2001;10:393-413.

47. American Psychiatric Association. Diagnostic and statistical manual of mental disorders (4th ed., text rev.). Washington, DC; 2000.

48. Leymann H, Gustafsson A. Mobbing at work and the development of posttraumatic stress disorders. Eur J Work Org Psychol 1996;5:251-75.

49. Rodríguez-Muñoz A, Moreno-Jiménez B, Sanz-Vergel AI, Garrosa E. Post-traumatic symptoms among victims of workplace bullying: Exploring gender differences and shattered assumptions. J Appl Soc Psychol. 2010;40:2616-35.

50. Matthiesen SB, Einarsen S. Psychiatric distress and symptoms of PTSD among victims of bullying at work. Br J Guid Counc 2004;32:335-56.

51. Janoff-Bulman R. Shattered assumptions-towards a new psychology of trauma. New York: The Free Press; 1992.

52. Ravin JM, Boal CK. Post-traumatic stress disorder in the work setting: Psychic injury, medical diagnosis, treatment and litigation. Am J Forensic Psychiatry 1989;10:5-23.

53. Hoel H, Einarsen S, Cooper CL. Organisational effects of bullying. En Einarsen S. Hoel H, Zapf D, Cooper CL (Eds.). Bullying and Emotional Abuse in the Workplace. International perspectives in research and practice. London: Taylor \& Francis; 2003.

54. Kivimäki M, Elovainio M, Vahtera J. Workplace bullying and sickness absence in hospital staff. Occup Environ Med 2000;57;656-60.

55. Rodríguez-Muñoz A, Baillien E, De Witte H, Moreno-Jiménez B, Pastor JC. Cross-lagged Relationships between Workplace Bullying, Job Satisfaction and Engagement: Two Longitudinal Studies. Work Stress 2009;23:225-43.

56. Einarsen S, Matthiesen SB, Skogstad A. Bullying, burnout and well-being among assistant nurses. J Occup Health Safety 1998;14:263-68.

57. Kivimäki M, Virtanen M, Vartia M, Elovainio M, Vahtera J, Keltikangas-Järvinen L. Workplace bullying and the risk of cardiovascular disease and depression. Occup Environ Med 2003;60:779-83.

58. Nielsen MB, Hetland J, Matthiesen SB, Einarsen S. Longitudinal relationships between workplace bullying and psychological distress Scand J Work Environ, in press.

59. Geurts SAE, Sonnentag S. Recovery as an explanatory mechanism in the relation between acute stress reactions and chronic health impairment. Scand J Work Environ Health 2006;32:482-92.

60. Hansen AM, Hogh A, Persson R, Karlson B, Garde AH, Orbaek P. Bullying at work, health outcomes, and physiological stress response. J Psychosom Res 2006;60:63-72.

61. Kudielka BM, Kern S. Cortisol day profiles in victims of mobbing (bullying at the work place): preliminary results of a first psychobiological field study. J Psychosom Res 2004;56:149-50. 
62. Moreno-Jiménez B, Rodríguez-Muñoz A, Sanz-Vergel AI, Moreno Y. Acoso psicológico en el trabajo y problemas de salud: El papel de la activación fisiológica. Psicothema 2011;23(2):227-32.

63. Melamed S, Shirom A, Toker S, Berliner S, Shapira I. Burnout and risk of cardiovascular disease: evidence, possible causal paths, and promising research directions. Psychol Bull 2006;132:327-53.

64. Escartín J, Arrieta C, Rodríguez-Carballeira A. Mobbing o Acoso laboral: Revisión de los principales aspectos teórico-metodológicos que dificultan su estudio. Actualidades en Psicología 2009;23(110):1-19.

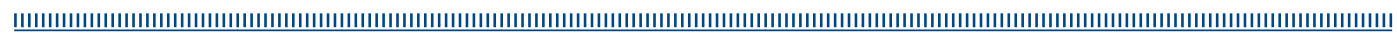

